\title{
Die schleichende Ausweitung der konkreten Gefährdungs- delikte und die damit einhergehende Verschärfung der polizeilichen Pflichten bei hilflosen Personen
}

\author{
Anmerkung zu BGH 3 StR 463/07-Urteil vom 10. Januar 2008: Aussetzung mit Todesfolge \\ durch Polizeibeamte; zugleich ein Beitrag zum Tatbestand der Aussetzung*
}

Sönke Gerhold

\section{Teil: Einleitung}

Haftstrafenvermeidung und Rechtsgüterschutz, das waren die Leitlinien der Großen Strafrechtsreform 1969 bis 1975, positive Spezialprävention das Ziel ${ }^{1}$. Der Blick war ganz auf den Täter gerichtet und seine Resozialisierung sollte im Vordergrund der strafrechtlichen Erwägungen stehen ${ }^{2}$. Doch was ist von diesen Forderungen noch übrig?

Im Strafrecht hat in den letzten Jahrzehnten ein Umdenken stattgefunden. Das Opfer ist mehr und mehr in den Mittelpunkt kriminologischer und strafrechtlicher Betrachtungen gerückt ${ }^{3}$. Der geänderte Blickwinkel, weg von einer rein täterorientierten Betrachtung hin zu einer mehr opferorientierten oder zumindest auch opferorientierten Betrachtung ${ }^{4}$, hat zahlreiche Gesetzesänderungen der jüngsten Zeit begünstigt. $\mathrm{Zu}$ denken ist allein an das Opferrechtsreformgesetz ${ }^{5}$ oder das zweite Justizmodernisierungsgesetz ${ }^{6}$, beides Gesetze, die sich ausdrücklich dem Opferschutz verschrieben haben. Die Nebenklage wurde nicht zuletzt durch die Erweiterung des \$397a I 1 StPO auf nahe Angehörige des Getöteten ${ }^{7}$ und ihre Einführung im Jugendstrafverfahren ${ }^{8}$ erheblich gestärkt. Das 2. Opferrechtsreformgesetz soll noch im Frühjahr 2009 auf den Weg gebracht werden und den Katalog des $\$ 395$ StPO erneut erweitern ${ }^{9}$. Die Tendenz, das Opfer mehr in den Mittelpunkt der Betrachtung zu rücken, setzt sich also weiter fort.

Deutlich wird dies auch in einer Presseerklärung der Bundesjustizministerin Zypries, die erklärt: „Die Bekämpfung von Kriminalität und Gewalt ist eine der wichtigsten Aufgaben des Staates. Allerdings dürfen wir nicht nur für die Bestrafung der Täter sorgen. Wir tragen auch Verantwortung für die Opfer einer Straftat. Dieser Aufgabe ist der Staat in den letzten Jahren ein gutes Stück gerecht geworden. Noch vor 20 Jahren ging es ganz überwiegend um die Täter - das Opfer spielte so gut wie keine Rolle. Heute weiß man, dass Verletzte Subjekte im Verfahren sind und eigene Rechte haben. Mit unserem Gesetzentwurf wollen wir den Schutz der Opfer und Zeugen von Straftaten noch weiter stärken. Vor allem für Opfer schwerer Straftaten ist es sehr wichtig, dass ihre Belange im Strafverfahren angemessen berücksichtigt werden. “10

Neben diesen und weiteren durchaus begrüßenswerten Aspekten des Opferschutzes kann aber auch eine weite, fast unverhältnismäßige und täterunfreundliche Auslegung einzelner Tatbestandsmerkmale oder eine unangemessene Kriminalisierung Folge des geänderten Blickwinkels und des regelmäßigen Rufs nach Erweiterungen und Verschärfungen des Strafrechts sein ${ }^{11}$. Verschiedentlich wurde bereits vor einer ausufernden Viktimodogmatik ${ }^{12}$ und Viktimagogie gewarnt ${ }^{13}$. Täter- und Opferbelange müssen daher stets umfassend gegeneinander abgewogen werden, um für beide Seiten gerechte Ergebnisse zu erzielen ${ }^{14}$. Eine wie auch immer geartete einseitige Betrachtung kann den widerstreitenden Interessen nicht gerecht werden. So anerkennenswert auch die von Reemtsma treffend gekennzeichneten Opferinteressen an einer Verurteilung des Täters $\operatorname{sind}^{15}$, so wenig darf die Strafmaßfindung aber übermäßig von Genugtuungsinteressen der Opfer beeinflusst werden ${ }^{16}$. Die schuldangemessene Strafe ist und bleibt der bestimmende und zugleich einzig gerechte Faktor. Von diesem Leitbild geführt, lassen sich sowohl zu harte, als auch zu milde Strafen bestimmen, zu denen eine rein täterorientierte Betrachtung unter Ausklammerung des Opfers und seiner Interessen führen kann.

Der vorliegende Fall zeichnet sich nun dadurch aus, dass das Verfahren gleich in mehrfacher Hinsicht durch opferorientierte Verfahrensrechte und eine entsprechend weite Auslegung der jeweiligen Tatbestandsmerkmale geprägt war. Die Folge einer solchen Auslegung für die betreffenden Beamten wurde nicht berücksichtigt und auch eine allgemeine Gerechtigkeitsprüfung, gemessen an der Schuld der Beamten, wird im folgenden Urteil vermisst.

Der tragische Tod des 18-jährigen Robert S., der am 01.12.2002 stark alkoholisiert an den Folgen eines Verkehrsunfalls verstarb, nachdem ihn zwei Polizeibeamte zuvor in ihrem Dienstwagen aus einer Wohnsiedlung, in der er vehement Zutritt zu einem Wohnhaus unbekannter Dritter begehrt hatte, herausgefahren und auf einer Landstraße abgesetzt hatten, gibt daher Anlass die herrschende Auslegung des Aussetzungstatbestands sowohl unter täter- als auch unter opferorientierten Blickwinkeln zu betrachten.

Dass das Verfahren zunächst von der Staatsanwaltschaft Lübeck nach $\$ 170$ II StPO eingestellt worden ist, macht den bestimmenden Einfluss der Eltern des Verstorbenen, der späteren Nebenkläger, in besonderem Maße deutlich. Auch eine Vorschaltebeschwerde der Eltern nach $\$ 172$ I 1 StPO beim Generalstaatsanwalt in Schleswig blieb zunächst ohne Erfolg.

Erst durch ein erfolgreich geführtes Klageerzwingungsverfahren vor dem Schleswig-Holsteinischen Oberlandesgericht nach $\$ 172$ II StPO schafften sie die Voraussetzung der späteren Verurteilung.

Am 31.05.2007 wurden die beiden Beamten dann schließlich vom Landgericht Lübeck unter dem Aktenzeichen 5 Js 32601/04 1 Ks 4/06 wegen fahrlässiger Tötung zu Freiheitsstrafen von jeweils 9 Monaten auf Bewährung verurteilt.

Erneut war es an den Eltern des Verstorbenen als Nebenkläger eine Aufhebung des Schuldspruchs vor dem BGH zu erwirken. Auf die Revision der Nebenklage nach $\$ 401$ StPO legte der BGH eine Verurteilung wegen Aussetzung mit Todesfolge nahe. Die Revisionen 
von Verteidigung und Staatsanwaltschaft mit dem Ziel eines Freispruchs wurden als unbegründet verworfen.

Da die Sachverhaltsschilderungen der Polizeibeamten in weiten Teilen erheblich von den Feststellungen des Landgerichts Lübeck und später des Landgerichts Kiel abweichen, werden im Folgenden die prozessualen Erkenntnisse der Tatrichter in Lübeck, die auch der Entscheidung des BGH zugrunde liegen, Ausgangspunkt der Erwägungen sein.

Die Beweiswürdigung soll ausdrücklich nicht den Hauptgegenstand des Beitrags bilden, so dass erforderliche Schlüsse an gegebener Stelle offen bzw. einer tatrichterlichen Bewertung überlassen bleiben.

\section{Teil: Das Urteil des BGH}

Sein Urteil vom 10. Januar 2008, Az: 3 StR 463/0 $7^{17}$, hat der BGH wie folgt begründet:

„Das Landgericht hat die Angeklagten jeweils wegen fahrlässiger Tötung zur Freiheitsstrafe von neun Monaten mit Aussetzung der Vollstreckung zur Bewährung verurteilt. Hiergegen wenden sich die Revisionen der Angeklagten und - zu deren Gunsten - auch die Revision der Staatsanwaltschaft mit sachlich-rechtlichen Beanstandungen. Diese Rechtsmittel bleiben ohne Erfolg. Die Eltern des Getöteten erstreben als Nebenkläger mit ihren Revisionen die Verurteilung der Angeklagten wegen Aussetzung mit Todesfolge ( $\$ 221$ Abs. 1 und 3 StGB). Dieses Rechtsmittel führt zur Aufhebung des angefochtenen Urteils mit den Feststellungen und Zurückverweisung der Sache zu neuer Verhandlung und Entscheidung.

\section{Das Landgericht hat Folgendes festgestellt:}

Am 1. Dezember 2002 gegen 2.45 Uhr verließ der später zu Tode gekommene 18-jährige Gymnasiast S. eine Diskothek, in der er zusammen mit Freunden im Verlaufe der Nacht soviel Alkohol getrunken hatte, dass eine ihm nach seinem Tod entnommene Blutprobe 1,99\%, eine Urinprobe 2,84 \% Alkohol enthielt. Am Oberkörper war er nur mit einem T-Shirt und einem dünnen Baumwollpullover bekleidet. Die Außentemperatur lag bei ca. 4 Grad Celsius.

Nachdem der Heranwachsende wenige Hundert Meter von der Diskothek entfernt bewusstlos und halb auf der Straße liegend von Polizeibeamten gefunden worden und nach einer Untersuchung durch herbeigerufene Rettungssanitäter wieder allein zurückgeblieben war, klingelte er am nahe gelegenen Haus der ihm unbekannten Eheleute B. und erklärte, er wolle in das Haus, er wohne dort, seine Eltern hätten es vor eineinhalb Stunden gekauft. Durch den mehrfach geäußerten Hinweis des Zeugen B., dass dies nicht der Fall sei, ließ er sich von der Vorstellung nicht abbringen, in diesem Haus zu wohnen. Weil der junge Mann weiterhin versuchte, in das Haus zu kommen, verständigte die Zeugin B. die Polizei. Der Beamte der Einsatzleitstelle Z. schickte daraufhin die Angeklagten zum Haus der Eheleute B. mit der Information, dass dort ein Jugendlicher an Türen und Fenstern randaliere. Auch teilte er ihnen zumindest mit, dass es im näheren Umfeld kurz vorher bereits einen Vorfall gegeben hatte.

Als die Angeklagten mit ihrem Streifenwagen bei den Eheleuten B. ankamen, stand S. vor dem Haus und telefonierte mit seinem Handy. Er hatte nun seinerseits bei der Einsatzleitstelle der Polizei angerufen und erklärt, dass er in sein Haus und dort schlafen wolle. Nachdem die Angeklagten von den Eheleuten B. über das Vorgefallene informiert worden waren und mit dem - sich dabei weiterhin desorientiert verhaltenden - Heranwachsenden gesprochen hatten, war ihnen klar, dass dieser alkoholisiert, örtlich und situativ nicht orientiert und - aus welchen Gründen auch immer - nicht im Vollbesitz seiner geistigen Kräfte war. Sie hatten auch gesehen, dass er keine Jacke trug, was bei den herrschenden Witterungsbedingungen bei einem längeren Aufenthalt im Freien notwendig gewesen wäre.

Der Angeklagte M. forderte S. schließlich auf, das Grundstück der Eheleute B. zu verlassen und sprach einen Platzverweis aus. Der Betroffene ging auch zunächst in Richtung der Diskothek, kehrte dann aber wieder zum Haus zurück. Auch die zunächst weggefahrenen Angeklagten waren umgekehrt. Nachdem der Heranwachsende über eine Absperrkette gestürzt war, führte ihn der Angeklagte M. mit den Worten: „Jetzt ist Schluss, Freundchen, du kommst jetzt mit und kannst dich ausnüchtern" zum Streifenwagen. In diesem Moment waren die Angeklagten entschlossen, S. in den Polizeigewahrsam nach R. zu bringen.

Nachdem der Heranwachsende in das Dienstfahrzeug eingestiegen war und die Angeklagten seine Personalien sowie seine aktuelle Wohnanschrift in L. festgestellt hatten, kamen die Angeklagten nunmehr zu der Ansicht, dass der Gymnasiast doch kein Fall für den Gewahrsam in ihrer Dienststelle sei. Sie wollten ihn aber vom Grundstück der Eheleute B. wegbringen. Sie entschlossen sich daher, den Heranwachsenden in den benachbarten polizeilichen Zuständigkeitsbereich zu schaffen, um den ausgesprochenen Platzverweis durchzusetzen und einen Störer los zu sein.

Die Angeklagten fuhren in Richtung L., ließen S. nach einer Fahrtstrecke von ca. zehn Kilometern hinter einem Ortsausgang etwa acht Kilometer vor L. aus dem Streifenwagen aussteigen und fuhren davon. S. ging zurück in die Richtung, aus der er mit den Angeklagten gekommen war. Er legte der Straße folgend eine Strecke von rund zwei Kilometern zurück und zog dabei die Schuhe sowie seine Strümpfe aus. Etwa eine Stunde später wurde er auf der Fahrbahn sitzend von einer für die herrschenden Sichtverhältnisse zu schnell fahrenden Pkw-Lenkerin angefahren und nach links auf den Grünstreifen geschleudert. Er verstarb unmittelbar darauf an den durch den Zusammenprall mit dem Pkw entstandenen schweren Verletzungen.

\section{Revision der Nebenkläger}

1. Die rechtliche Würdigung der getroffenen Feststellungen begegnet - entgegen der Auffassung des Generalbundesanwalts - durchgreifenden rechtlichen Bedenken, soweit das Landgericht eine Ausset-

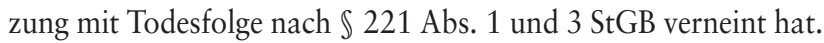

a) Dies hat das Landgericht damit begründet, dass bereits Zweifel bestünden, ob die Angeklagten S. objektiv in eine hilflose Lage versetzt bzw. ihn in einer solchen im Stich gelassen haben. Auch wenn der Heranwachsende geistig beeinträchtigt gewesen sei, habe er sein Handy bei sich gehabt und sei jedenfalls teilweise in der Lage gewesen, damit ordnungsgemäß umzugehen. Er sei auch in der Lage gewesen, sich zu artikulieren und sich fortzubewegen.

Jedenfalls habe die Kammer nicht mit ausreichender Sicherheit feststellen können, dass die Angeklagten in Bezug auf eine hilflose Lage zumindest bedingt vorsätzlich gehandelt hätten. Sie hätten das Ausmaß seiner Alkoholisierung nicht erkannt und die sonstigen Anzeichen für die Beeinträchtigung seines geistigen Zustandes nicht ausreichend gewürdigt.

b) Dies hält rechtlicher Nachprüfung nicht stand. Nach den Urteilsfeststellungen befand sich S. jedenfalls nach dem Verlassen des Streifenwagens in einer hilflosen Lage im Sinne von $\$ 221$ Abs. 1 StGB. 
In einer solchen ist, wer der abstrakten Gefahr des Todes oder einer schweren Gesundheitsbeschädigung ohne die Möglichkeit eigener oder fremder Hilfe ausgesetzt ist (vgl. Hardtung in MünchKomm, \221 Rdn. 7). Hilflosigkeit im Sinne des Tatbestandes definiert sich danach als das Fehlen hypothetisch rettungsgeeigneter sächlicher Faktoren oder hilfsfähiger (und generell auch hilfsbereiter) Personen (vgl. Horn/Wolters in SK-StGB 54. Lfg. \$ 221 Rdn. 3). Eine derartige Lage war hier mit Blick auf die Feststellungen zu den äußeren Umständen und dem Verhalten des Heranwachsenden nach dem Verlassen des Streifenwagens ganz offensichtlich gegeben. Der Umstand, dass der junge Mann ein funktionstüchtiges Handy bei sich trug, das er nach dem Aussteigen zumindest zeitweise auch bedienen konnte, ändert hier an der Annahme einer objektiv hilflosen Lage im Sinne des $\mathbb{2} 21 \mathrm{StGB}$ schon deshalb nichts, weil es ihm nicht gelungen ist, jemanden anzurufen und er zudem gar nicht wusste, wo er sich befand.

c) Die fehlerhafte rechtliche Beurteilung des objektiven Tatbestandes hat zur Folge, dass auch der durch das Landgericht vorgenommenen Würdigung der subjektiven Tatseite die Grundlage entzogen ist. Im Übrigen steht die Begründung, mit der das Landgericht ein (zumindest bedingt) vorsätzliches Handeln der Angeklagten abgelehnt hat, nicht im Einklang mit den getroffenen Feststellungen. Danach haben die Angeklagten die Alkoholisierung des Heranwachsenden ebenso erkannt, wie den Umstand, dass er - aus welchen Gründen auch immer - nicht im Vollbesitz seiner geistigen Kräfte war. Dass die Angeklagten die vorhandene starke Alkoholisierung nicht zutreffend, sondern geringer eingeschätzt haben, ist daher ohne Belang.

Aus welchen Gründen sich die Angeklagten dann aber der konkreten Gefährdung des Lebens und der Gesundheit des Heranwachsenden nicht bewusst gewesen sein sollten, ist nicht ersichtlich. Dass die Angeklagten die Durchführung des Verbringungsgewahrsams und dessen Ausgang entgegen der bestehenden Dienstanweisung der Einsatzleitstelle nicht bzw. nicht vollständig gemeldet haben, könnte vielmehr für das Bewusstsein der Angeklagten sprechen, dass ihr Verhalten zu einer bedrohlichen Verschlechterung der Lage des Hilfsbedürftigen führen werde oder zumindest führen könnte (vgl. BGH NStZ 1985, 501; Fischer, StGB 55. Aufl. \$ 221 Rdn. 12).

2. Die aufgezeigten Rechtsfehler führen auf die Revision der Nebenkläger zur Aufhebung des angefochtenen Urteils. Die Sache bedarf neuer Verhandlung und Entscheidung. Der Senat hat von $\mathbb{} 354$ Abs. 2 Satz 1 2. Alt. StPO Gebrauch gemacht.

\section{Revisionen der Angeklagten und der Staatsanwaltschaft}

Die Revisionen der Angeklagten und das - zu deren Gunsten eingelegte - Rechtsmittel der Staatsanwaltschaft, das der Generalbundesanwalt entgegen seiner Antragsschrift in der Revisionshauptverhandlung nicht mehr vertreten hat, sind offensichtlich unbegründet.

1. Zum Schuldspruch zeigen die Revisionen keinen Rechtsfehler auf.

Die Angeklagten haben den Tod des Heranwachsenden im Sinne des \$2 22 StGB durch Fahrlässigkeit verursacht, indem sie diesen unter den gegebenen Umständen, insbesondere trotz seines von ihnen erkannten geistigen Zustandes und der widrigen äußeren Verhältnisse aus ihrem Zuständigkeitsbereich verbracht und nach einer Fahrtstrecke von zehn Kilometern aus dem Streifenwagen haben aussteigen lassen.

a) Die Angeklagten haben den Tod von S. verursacht. Das verkehrsordnungswidrige Verhalten der Unfallverursacherin hat den not- wendigen Zusammenhang zwischen dem Verhalten der Angeklagten und dem Tode von S. nicht unterbrochen. Es ist anerkannt, dass eine Ursache im Rechtssinne ihre Bedeutung nicht verliert, wenn außer ihr noch andere Ursachen zur Herbeiführung des Erfolges beitragen. Ein Ursachenzusammenhang ist nur dann zu verneinen, wenn ein späteres Ereignis die Fortwirkung der ursprünglichen Bedingung beseitigt und seinerseits allein unter Eröffnung einer neuen Ursachenreihe den Erfolg herbeigeführt hat (vgl. BGHSt 39, 322, 324 m.w.N.; BGH NStZ 1994, 83). Dies war hier ersichtlich nicht der Fall. Vielmehr schloss sich die von der Unfallfahrerin gesetzte Ursachenreihe unmittelbar an das pflichtwidrige Verhalten der Angeklagten an und baute auf diesem auf. Die ursprüngliche Bedingung war nicht beseitigt, sondern wirkte fort.

b) Die Angeklagten haben im Hinblick auf den Tod von S. auch fahrlässig gehandelt. Gerade die Durchführung eines so genannten Verbringungsgewahrsams und die Entlassung des Heranwachsenden unter den gegebenen Umständen hat objektiv und subjektiv vorhersehbar zu dessen Tod geführt. Die Einzelheiten des durch das Verhalten in Gang gesetzten Verlaufs brauchen nicht vorhersehbar sein. Tritt der Erfolg durch das Zusammenwirken mehrerer Umstände ein, müssen dem Täter alle erkennbar sein, weil nur dann der Erfolg für ihn voraussehbar ist (vgl. BGH NStZ 2001, 143, 145; NStZ 2004, 151; Jähnke in LK 11. Aufl. $\$ 222$ Rdn. 3 m.w.N.). Dass sich der betrunkene und desorientierte Heranwachsende bei der herrschenden Dunkelheit an einer Straße orientiert und sich auf die Fahrbahn begibt, dort von einem Auto erfasst wird und an den Folgen eines solchen Verkehrsunfalls verstirbt, lag unter den gegebenen Umständen nach dem Maßstab des gewöhnlichen Erfahrungsbereiches (vgl. BGHSt 12, 75, 78) nahe. Dies war daher objektiv sowie für die Angeklagten als erfahrene Polizeibeamte, die mit den Verhaltensweisen von Betrunkenen und den Gefahren des nächtlichen Straßenverkehrs vertraut waren, auch subjektiv vorhersehbar.

Daran ändert sich nichts dadurch, dass das Sitzen des Heranwachsenden auf der Fahrbahn, insbesondere bei der herrschenden Dunkelheit, unvernünftig war. Zwar kann eine gänzlich vernunftswidrige Handlungsweise eines Getöteten die Vorhersehbarkeit des Erfolgs entfallen lassen (vgl. BGHSt 3, 218; 4, 182, 187; 12, 75, 78). Allerdings entfällt die Vorhersehbarkeit in solchen Fällen nur dann, sofern der Getötete entscheidungsfähig, insbesondere nicht etwa betrunken war (vgl. Jähnke in LK 11. Aufl. \$ 222 Rdn. 9). S. war indes nicht nur betrunken, sondern in engem zeitlichen Zusammenhang mit seinem Aussteigen aus dem Streifenwagen örtlich sowie situativ desorientiert und damit in seinen geistigen Leistungen deutlich vermindert gewesen. Danach war er offensichtlich nicht entscheidungsfähig. Da die Angeklagten dies erkannt hatten, kann ihre strafrechtliche Haftung nicht wegen des objektiv unvernünftigen Verhaltens des Heranwachsenden entfallen. Auch der Umstand, dass ein anderer Verkehrsteilnehmer sich - wie hier - nicht pflichtgemäß verhält, sondern unter Verstoß gegen die Straßenverkehrsordnung zu schnell fährt, war als alltägliches Geschehen nahe liegend und daher vorhersehbar.

2. Auch die Überprüfung des Strafausspruchs aufgrund der Revisionsrechtfertigungen hat im Ergebnis keinen Rechtsfehler zum Nachteil der Angeklagten ergeben. “

\section{Teil: Zur Aussetzung mit Todesfolge}

Das Landgericht Kiel hat den Angeklagten M durch Urteil vom 17.09.2008 nach den Vorgaben des BGH unter dem Aktenzeichen 
8 Ks 6/08 ${ }^{18}$ wegen Aussetzung mit Todesfolge in einem minder schweren Fall zu einer Freiheitsstrafe von 1 Jahr und 6 Monaten verurteilt, den Angeklagten $G$ wegen fahrlässiger Tötung zu einer Freiheitsstrafe von 9 Monaten. Beide Strafen setzte das Landgericht Kiel zur Bewährung aus.

Die differenzierende Betrachtung hinsichtlich M und G beruht auf einer im Verhältnis zum Landgericht Lübeck abweichenden Sachverhaltsfeststellung, nach der G den Zustand des S im Gegensatz zu $M$ nicht richtig einzuschätzen vermochte oder ihm zumindest keine Kenntnis der fehlenden Orientierung des $\mathrm{S}$ nachzuweisen war.

Die Verurteilung wegen fahrlässiger Tötung unterliegt auch aus der Warte eines objektiven Betrachters keinen durchgreifenden Bedenken. Dem BGH ist insoweit zuzustimmen. Zur Begründung verweise ich auf die folgende Darstellung, der sich diese unmittelbar entnehmen lässt.

Anders verhält es sich jedoch mit der Verurteilung zur Aussetzung mit Todesfolge. Die hierauf bezogenen Ausführungen bilden den Schwerpunkt des Beitrags.

\section{A. Strafbarkeit wegen Aussetzung mit Todesfolge gemäß \ 221 I Nr. 1, III StGB durch die Aufforderung an S das Auto zu verlassen}

Sofern man an eine Aussetzung mit Todesfolge gemäß $\$ 221$ I Nr. 1, III StGB hinsichtlich der Aufforderung an S, den Polizeiwagen zu verlassen, denkt, stellt sich zunächst die Frage, ob überhaupt der Grundtatbestand verwirklicht wurde. Wenn man die oben abgedruckte Entscheidung studiert, fällt einem auf, dass der BGH sich auf Ausführungen zum Grundtatbestand beschränkt. Auf die Voraussetzungen der Erfolgsqualifikation geht er mit keinem Wort ein. Es sollen daher zunächst die Erwägungen hinsichtlich des Grundtatbestands auf ihre Richtigkeit überprüft werden

\section{Grundsätzliche Erwägungen in Bezug auf den Grundtatbestand}

\section{Hilfslose Lage}

Der BGH bejaht die hilflose Lage im Ergebnis zutreffend. S war vorliegend mit einer Blutalkoholkonzentration von mindestens 1,99\%o objektiv schwer alkoholisiert und zudem örtlich und situativ nicht orientiert, was nicht zuletzt aus dem Umstand folgt, dass er, jeglichem Argument unzugänglich, gemeint hat, in dem Haus der Eheleute B zu wohnen. Darüber hinaus konnte er auch sein Handy nicht mehr sicher bedienen und die Möglichkeit über das Handy Hilfe zu holen, war wegen der späten Stunde erheblich eingeschränkt. Er war gegen die niedrigen Temperaturen unzureichend geschützt und unterlag auf Grund seiner Alkoholisierung der Gefahr, sich selbst zu verletzen oder zu gefährden.

Dennoch möchte ich diesen Beitrag zum Anlass nehmen, ein paar weiterführende Anmerkungen zur hilflosen Lage im Tatbestand der Aussetzung zu machen, die sich unmittelbar an das dem Beitrag übergeordnete Thema eines schuldangemessenen Umgangs mit dem geltenden Strafrecht anschließen. Es soll gezeigt werden, wie eine restriktive Auslegung des Aussetzungstatbestands erreicht werden kann und warum eine solche restriktive Auslegung dringend geboten ist.

\section{a. Die hilflose Lage, ein aliud zur konkreten Gefahr?}

Richtigerweise ist die hilflose Lage ein aliud zur konkreten Gefahr des Todes oder der schweren Gesundheitsschädigung, wie sie $\mathbb{} 221$ I StGB a.E. fordert ${ }^{19}$. Diese begriffliche Trennung folgt bereits aus dem Wortlaut des $\mathbb{2} 21$ I StGB, der durch die Verwendung des Begriffs „dadurch“ zum Ausdruck bringt, dass entsprechend den \\} 3 1 5 \text { ff. StGB nicht jede konkrete Gefährdung eines anderen Men- } schen unter Strafe gestellt sein soll, sondern nur eine solche, die ihren spezifischen Ursprung in der hilflosen Lage selbst hat. Darüber hinaus wird dieses Wortlautargument auch durch einige wesentliche systematische Erwägungen gestützt.

Ebel führt in einem sehr lehrreichen Beitrag zur hilflosen Lage im Straftatbestand der Aussetzung aus, verstehe man \$221 I StGB als allgemeines Gefährdungsdelikt ohne Erfordernis einer näher beschriebenen Tathandlung, wären die $\mathbb{S}$ 315a bis c StGB in ihrem Grundfall Privilegierungen zum Aussetzungstatbestand ${ }^{20}$. Dies folge bereits aus dem geringeren Strafmaß. Es sei jedoch keinesfalls zu begründen, warum ein festgeschriebenes, zweites Unrechtselement - das beispielsweise im Falle des $\ 316$ StGB selbständig unter Strafe gestellt ist $\mathrm{t}^{21}$ - die Strafe geringer ausfallen lasse, als ein sonstiger unbestimmter Fall der konkreten Gefährdung. Ein solches Verständnis überschreite die Grenze zulässiger Auslegung. Darüber hinaus werde auch der Vollendungszeitpunkt bedenklich weit nach vorne verlagert, wenn man die Begriffe der hilflosen Lage und der Gefahr für Leib und Leben gleichsetze.

Dem ist ohne Einschränkung zuzustimmen.

\section{b. Die Begriffsbestimmung der herrschenden Meinung}

Die hilflose Lage muss damit erst zu einer konkreten Gefahr führen, für ihre Begründung genügt folglich ein Weniger. Es befindet sich daher nach herrschender Definition derjenige in einer hilflosen Lage, der der abstrakten Gefahr des Todes oder einer schweren Gesundheitsschädigung ohne die Möglichkeit eigener oder fremder Hilfe ausgesetzt ist ${ }^{22}$. Der Begriff der abstrakten Gefahr meint dabei mehr, als eine bloß potentielle oder hypothetische Gefahr ${ }^{23}$. Es muss sich um eine reale Gefahr für Leib und Leben handeln, deren Eintritt jedoch noch nicht beachtlich wahrscheinlich ist, sondern für den eine geringe Wahrscheinlichkeit genügt ${ }^{24}$. Ohne eigene oder fremde Hilfe ist, wem zur Abwendung der Gefahr, kennt er sie oder hätte er sie erkannt, weder die Hilfe des eigenen Körpers, einer Sache, eines Tieres oder eines anderen Menschen zur Verfügung steht, wobei die Hilfe eines Dritten Hilfsfähigkeit und Hilfswilligkeit desselben voraussetzt $^{25}$. Abzugrenzen ist die Hilflosigkeit demnach von der bloßen Ahnungslosigkeit ${ }^{26}$. Zur Prüfung der Hilflosigkeit muss die Kenntnis der Gefahr unterstellt werden ${ }^{27}$.

\section{c. Weitergehende Anforderungen}

Durch diese weit verbreitete Definition der hilflosen Lage ist allerdings noch nichts über das zeitliche Verhältnis zwischen hilfloser Lage und konkreter Gefahr oder die Art der Gefahr gesagt. Es stellt sich daher die weiterführende Frage, ob die hilflose Lage der konkreten Gefahr immer zeitlich vorausgehen muss bzw. eine gewisse Dauer voraussetzt oder ob sie auch zeitlich mit der konkreten Gefährdung zusammen fallen kann, sowie die Frage, ob jede denkbare Gefahr den Tatbestand des $\$ 221$ I Nr. 1 StGB eröffnet. 


\section{(1) Die neuere Rechtsprechung zu $\mathbb{\int} 315 b$ I StGB}

Im Rahmen des gefährlichen Eingriffs in den Straßenverkehr nach \315b I StGB hat der BGH lange Zeit die Auffassung vertreten, dass es zur Tatbestandsverwirklichung nicht genüge, wenn der Eingriff des Täters unmittelbar zu einer konkreten Gefahr führe, ohne dass diese auf einem durch den Eingriff beeinträchtigten Verkehrsablauf beruhe ${ }^{28}$. Erschöpfe sich der Eingriff in der Gefährdung bzw. Verletzung des Opfers, scheide ein gefährlicher Eingriff in den Straßenverkehr aus ${ }^{29}$.

Von dieser Rechtsprechung ist der 4. Senat des BGH in BGHSt 48, 119 ff. allerdings zumindest für den Fall abgerückt, dass im fließenden Verkehr Gegenstände auf Fahrzeuge geworfen werden und die Tathandlung deshalb unmittelbar zu einem bedeutenden Fremdschaden führt, solange sich die unmittelbare Schädigung als Steigerung der durch die Tathandlung bewirkten abstrakten Gefahr für die Sicherheit des Straßenverkehrs darstelle.

An der Voraussetzung der abstrakten, verkehrsspezifischen Gefahr wird damit ausdrücklich festgehalten. Lediglich die zeitliche Komponente wird für gänzlich entbehrlich erachtet. So heißt es in der Entscheidung: „Erforderlich ist, dass die Tathandlung eine abstrakte Gefahr für die Sicherheit des Straßenverkehrs bewirkt, die sich zu einer konkreten Gefahr für die genannten Schutzobjekte verdichtet. Das Erfordernis einer zeitlichen Differenz zwischen Eingriff und konkreter Gefahr ist dem Wortlaut der Vorschrift dagegen nicht zu entnehmen. Der Tatbestand des $\$ 315$ b Abs. 1 StGB kann daher in sämtlichen Handlungsalternativen auch dann erfüllt sein, wenn die Tathandlung unmittelbar zu einer konkreten Gefahr oder Schädigung führt, sofern sich dieser Erfolg als Steigerung der abstrakten Gefahr darstellt. Auch im Blick auf das in $\ 315$ b StGB geschützte Rechtsgut, die Sicherheit des Straßenverkehrs, die ohne die Notwendigkeit einer Gemeingefahr den Schutz von Individualrechtsgütern wie Leben, Gesundheit und bedeutende Sachwerte mitumfasst, besteht kein Anlass, zwischen zeitlich gestreckten und auf Minutenbruchteile reduzierten Geschehensabläufen zu unterscheiden." Die weitergehende Argumentation der Entscheidung überzeugt.

Im Rahmen des $\ 315$ b I StGB kann es daher als gesicherte Erkenntnis angesehen werden, dass es keiner Perpetuierung der abstrakten Gefahrenlage bedarf.

\section{(2) Die Übertragbarkeit auf den Aussetzungstatbestand}

Fraglich ist nun, ob sich diese Erkenntnis auf den Tatbestand der Aussetzung übertragen lässt. Der Tatbestand der Aussetzung spielt in der Praxis nur eine sehr untergeordnete Rolle ${ }^{30}$ und wird in der polizeilichen Kriminalstatistik nach wie vor nicht gesondert ausgewiesen. Die geringe Zahl von Anklagen und Verurteilungen führt dazu, dass die Rechtsprechung nur wenig Gelegenheit hat, sich fortzuentwickeln und alte Auffassungen zu revidieren und zu überdenken; ganz im Gegensatz zu den strukturgleichen Straßenverkehrsdelikten. In vielen Aussetzungsfällen orientiert sich die Rechtsprechung deshalb an den im Rahmen der Straßenverkehrsdelikte entwickelten Grundsätzen.

\section{(a) Die aussetzungsspezifische Gefahr als tatbestandliches Korrektiv}

Der Tatbestand des $\ 315 \mathrm{~b}$ I StGB wird nun dadurch eingeschränkt, dass nur verkehrsspezifische Gefahren, die auf für Verkehrsvorgänge typische Fortbewegungskräfte zurückzuführen sind, Berücksichti- gung finden - so die Eigendynamik des vom Täter selbst benutzten Fahrzeugs, die Fremddynamik eines von einem anderen Verkehrsteilnehmer genutzten Fahrzeugs oder das Zusammenwirken beider Kräfte.

Will man die Entscheidung des 4. Senats auf den Aussetzungstatbestand übertragen, zweifelt man zunächst, ob es entsprechend den Verkehrsgefahren überhaupt aussetzungs(-tatbestands-)spezifische Gefahren gibt. Schützt $\ 221$ StGB doch gerade Leib und Leben von Personen in hilfloser Lage vor den verschiedensten Gefahren. Dies können anerkanntermaßen typische Verkehrsgefahren sein, wie im vorliegenden Fall das Überfahrenwerden, aber auch Naturgefahren, wie Erfrieren, oder sonstige Gefahren, wie Opfer vorsätzlicher Straftaten Dritter zu werden.

Gemein ist diesen Gefahren allerdings, dass sie vom Täter in der konkreten Aussetzungssituation nicht beherrscht werden können und ihre generelle Existenz auch grundsätzlich nicht auf den Täter zurückzuführen ist, so dass der Zeitpunkt ihres Eintritts ungewiss bleibt. Der Täter wirkt lediglich auf das Aussetzungsopfer oder die möglichen Hilfsmittel ein. Die Gefahr selbst ist dann eine ausschließlich mittelbare Folge der Hilflosigkeit, auf die der Täter aber keinen Einfluss hat. Zu ihrer Abwendung kann er nicht die Gefahr beeinflussen, sondern lediglich Rettungsmaßnahmen ergreifen.

Hat der Täter in seltenen Fällen die Ursache der Gefahr selbst gesetzt, muss die potentielle Gefahrenlage schon vor der Aussetzungshandlung bestanden haben, um mit den sonstigen Aussetzungsgefahren vergleichbar zu sein.

Eine aussetzungstypische Gefahr ist daher nur eine solche, die bereits vor der Aussetzungshandlung bestand oder ihre Ursache nicht in einem Verhalten des Täters hat, sondern ganz allgemein droht, wenn ein Mensch in einer bestimmten Lage keine Abwehrmaßnahmen ergreift, sei es auch nur durch warme Kleidung, Proviant oder die eigene Sorgfalt im Umgang mit einer Gefahrenquelle oder einer gefährlichen Situation.

Da Tathandlung und Gefahr strikt getrennt werden müssen ${ }^{31}$, ist es also erforderlich, dass der Täter einen bestehenden Mangel oder eine bestehende und für den Täter in der Aussetzungssituation unbeherrschbare Gefahr ausnutzt.

In der Beschränkung des Tatbestands auf aussetzungstypische Gefahren liegt zugleich die Antwort auf die von Fischer aufgeworfene Frage, wie die bloße Verursachung von Gefahren vom Tatbestand ausgenommen werden könne ${ }^{32}$. Auch Hardtung grenzt in Randnummer 12 in ähnlicher Weise die aussetzungsspezifische Gefahr von sonstigen Gefahren $\mathrm{ab}^{33}$.

Durch eine so verstandene Auslegung werden zugleich andere Verletzungs- und Gefährdungsdelikte vom Tatbestand des $\ 221$ StGB ausgenommen, ohne dass er seine Funktion als Auffangtatbestand gerade für nicht speziell geregelte Gefahren verlöre. Die tatbestandliche Abgrenzung zu sonstigen Verletzungs- und Gefährdungsdelikten ist damit Folge einer durch die hohe Strafdrohung gebotenen engen Auslegung, nicht ihr Grund ${ }^{34}$.

Ein Totschlag durch den Schuss aus einer Pistole oder ein gefährlicher Eingriff in den Straßenverkehr durch das Werfen eines Steins verwirklicht daher nicht stets zugleich den Tatbestand des $\mathbb{} 221$ StGB, auch wenn schwerlich bezweifelt werden kann, dass sich ein Mensch, auf den geschossen oder auf dessen Auto ein Stein geworfen wird, nicht der unmittelbar folgenden Verletzung hilflos gegenüber sieht. Auch wenn der Täter sein Opfer unmittelbar vor ein her- 
annahendes Auto stößt, verwirklicht sich keine aussetzungstypische Gefahr, da der Täter das Geschehen in diesem Fall beherrscht und den Erfolg unmittelbar herbeiführt. Im Ergebnis muss daher ein wenn auch noch so kleiner Raum für Zufälle ${ }^{35}$ verbleiben, um von einer aussetzungsspezifischen Gefahr sprechen zu können, eine längere zeitliche Differenz zwischen der abstrakten Gefahr und ihrer Zuspitzung jedoch nicht. Die konkrete Gefahr des Kälteschocks eines Ausgesetzten kann daher sofort oder erst nach einiger Zeit eintreten.

Die Grenzen des Wortlauts dürften durch diese Auslegung ebenfalls nicht überschritten sein. Ob im Falle der Verwirklichung einer aussetzungsuntypischen Gefahr $\$ 221$ I Nr. 2 StGB durch das Imstichlassen verwirklicht werden kann, soll vorliegend nicht geklärt wer$\operatorname{den}^{36}$.

\section{(b) Die Perpetuierung der hilflosen Lage als tatbestandli- ches Korrektiv}

Ein entsprechendes Ergebnis versuchen Sternberg-Lieben/Fisch durch das Erfordernis einer Perpetuierung der hilflosen Lage zu erzielen $^{37}$, was auf den ersten Blick ebenfalls gut vertretbar erscheint. Für diese Auffassung streitet insbesondere der Wortlaut der gegenüber $\mathbb{3} 315 \mathrm{~b}$ StGB unmittelbar auf eine besondere „Lage“ abstellt. Aus dem Begriff der „Lage“ kann durchaus geschlossen werden, dass diese eine gewisse Stabilisierung erfordert und damit mehr als einen hilflosen Moment voraussetzt, in dem eine Rettung bereits aus zeitlichen Gründen ausgeschlossen ist.

Voraussetzung für eine hilflose Lage wäre dann, dass der Hilfsbedürftige die Realisierung der Gefahr bei Kenntnis der Gefahrenlage noch abwenden könnte oder dass Dritte sie noch abwenden könnten. Nutzt der Täter aber lediglich ein Überraschungsmoment zu einer Tötung, Körperverletzung oder zu einer sonstigen Tat, kann die fehlende Zeitspanne, um Abwehrmaßnahmen zu ergreifen, keine hilflose Lage begründen. Die Anforderungen an die Festigkeit und Dauerhaftigkeit der hilflosen Lage dürften aber keinesfalls überspannt werden.

Die Forderung nach einer perpetuierten hilflosen Lage führt jedoch in Grenzfällen anders als die Differenzierung nach aussetzungstypischen und aussetzungsuntypischen Gefahren zu Strafbarkeitslücken. Diese entstehen gerade in den Fällen, in denen eine Situation von solcher Gefährlichkeit ist, dass der Ausgesetzte von vornherein konkret gefährdet und das Unrecht damit gesteigert ist. Die Rettungschancen sind deutlich herabgesetzt. Anhaltspunkte dafür, dass der erhöhte Mindeststrafrahmen die über einen längeren Zeitraum anhaltende psychisch belastende Situation des Opfers mit all seinen Ängsten und Zweifeln hinsichtlich eines guten Ausgangs unter Strafe stellen will, fehlen ${ }^{38}$. Eine größere Gefahr muss daher unter Opferschutz- und Wertungsgesichtpunkten die geringere Gefahr mitumfassen.

\section{(c) Zwischenergebnis}

Im Hinblick auf die Weite des Tatbestands und die zugleich im Mindestmaß erhöhte Strafdrohung ist eine einschränkende Auslegung unabhängig vom Ansatzpunkt dringend geboten ${ }^{39}$. Keine oder nur Gefahren aus dem Tatbestand auszunehmen, die schlechterdings wegen ihrer kurzen Dauer nicht wahrgenommen werden können bevor der Erfolg eintritt oder die Realisierung ausgeblieben ist, erscheint zu kurz gegriffen ${ }^{40}$. Die so bezeichneten Augenblicksgefahren stellen zwar in keinem Fall aussetzungsspezifische Gefahren dar. Es sind jedoch nicht die einzigen aussetzungsuntypischen Gefahren. \221
StGB als allgemeines Lebens- und Gesundheitsgefährdungsdelikt zu begreifen ${ }^{41}$, ist mit dem im Mindestmaß erhöhten Strafrahmen unvereinbar. Dem Tatbestand muss eine gewisse Kontur verliehen werden.

Die Richtigkeit dieser Erwägungen belegt auch ein Strafrahmenvergleich mit den $\mathbb{\int} 223 \mathrm{ff}$. StGB.

Verletzt der Täter sein Opfer im Rahmen einer Körperverletzung schwer, beträgt der Strafrahmen Freiheitsstrafe bis zu fünf Jahren oder Geldstrafe. Selbst wenn die Verletzungen erst später zu einer schweren Gesundheitsschädigung führen, weil der Verletzte nicht schnell genug ärztlich versorgt wird, kann hierin kein Verbrechen der Aussetzung nach $\$ 221$ II Nr. 2 StGB gesehen werden, da sonst die Wertung des $\$ 226$ StGB umgangen würde. Es soll nämlich nicht jede schwere Gesundheitsschädigung dem Verbrechenstatbestand des $\$ 226$ StGB unterfallen, sondern nur einige besonders gewichtige Verletzungen. \$ 221 II Nr. 2 StGB lässt demgegenüber jede schwere Gesundheitsschädigung genügen.

Auch darf die Gefährdung nicht über $\$ 221 \mathrm{StGB}$ stärker bestraft werden, als ihre Realisierung. Die Gefahr der schweren Gesundheitsschädigung könnte nämlich gemäß $\$ 221 \mathrm{I}$ StGB nicht mit Freiheitsstrafe unter 3 Monaten bestraft werden. Die Realisierung einer schweren Gesundheitsschädigung unterfällt dagegen nur dem Grundtatbestand der Körperverletzungen, \$223 I StGB, der Freiheitsstrafe bis zu 5 Jahren oder Geldstrafe vorsieht.

\section{Kausales Versetzen in die hilflose Lage/Abgrenzung aktives Tun oder Unterlassen}

Auch ein Versetzen in die hilflose Lage wird man wohl mit dem $\mathrm{BGH}$ annehmen müssen. Entscheidend ist hier die Abgrenzung zwischen dem Versetzen durch aktives Tun und dem Im-Stich-Lassen des Abs. 1 Nr. 2. Ausführungen hierzu enthält das BGH-Urteil nicht, auch wenn die Abgrenzung nicht so klar auf der Hand liegt und weit reichende Konsequenzen hat.

Versetzen meint nach überwiegender Auffassung das Hervorrufen oder Steigern einer hilflosen Lage ${ }^{42}$. Befand sich der Ausgesetzte bereits zuvor in einer vergleichbaren hilflosen Lage, ist es erforderlich, dass die hilflose Lage durch die Täterhandlung zwischenzeitlich aufgehoben war $^{43}$.

Im vorliegenden Fall könnte der strafrechtliche Vorwurf zum einen an die Aufforderung, aus dem Auto auszusteigen, geknüpft werden, zum anderen an das Nicht-Nachhausefahren oder Nicht-In-DieAusnüchterungszelle-Verbringen, wie die Beamten es ursprünglich beabsichtigten.

Nach der herrschenden Lehre vom sozialen Handlungssinn ist der Schwerpunkt der Vorwerfbarkeit des strafrechtlich relevanten Verhaltens bei normativer Betrachtung und unter Berücksichtigung des sozialen Handlungssinns maßgeblich ${ }^{44}$. M und $G$ hätten $S$ an vielen geeigneten Orten aussteigen lassen können, der Polizeigewahrsam war nicht die einzige Möglichkeit die Gefahren von S abzuwenden. Hätten die Beamten jegliches weitere Tun unterlassen, wäre S im Polizeiwagen vor drohenden Gefahren geschützt gewesen.

$S$ allerdings in einem Zustand örtlicher und situativer Verwirrtheit aus dem Wagen zu weisen, eröffnete erst von neuem die Gefahren, die $S$ bereits zuvor gedroht, durch die angedachte Ingewahrsamnahme aber abgewendet worden waren.

Die hilflose Lage war durch die Täterhandlung zwischenzeitlich aufgehoben, weil die Beamten zunächst tatsächlich beabsichtigten, 
S in eine Ausnüchterungszelle zu bringen und ihn so vor Gefahren zu schützen ${ }^{45}$.

Der Schwerpunkt der Vorwerfbarkeit liegt demnach auf einem aktiven Tun.

Auch nach der alten Rechtslage vor dem 6. StrRG wurde das Hinausweisen einer unangemessen bekleideten und betrunkenen Person aus einer warmen Umgebung ins Freie trotz geringer Außentemperaturen als aktive Aussetzungshandlung aufgefasst ${ }^{46}$. Die Aussetzung ist in der Form des Begehungsdelikts zu prüfen.

Die hilflose Lage wurde auch kausal durch die Aufforderung auszusteigen herbeigeführt und ist den Beamten objektiv zurechenbar ${ }^{47}$. Dies folgt nicht zuletzt daraus, dass $S$ sich nicht freiverantwortlich selbst gefährdet hat. Er konnte aufgrund seiner örtlichen und situativen Desorientierung keine freiverantwortliche Entscheidung treffen und die drohenden Gefahren nicht erkennen. Die Beamten hatten ein überlegenes Gefährdungswissen.

Wichtig im Hinblick auf einen verantwortungsbewussten Umgang mit dem Aussetzungstatbestand ist allein, zu erkennen, dass im Rahmen des Grundtatbestands zweimal Kausalitäts- und Zurechnungserwägungen angestellt werden müssen. Einmal zwischen der Versetzungshandlung und der hilflosen Lage und einmal zwischen der hilflosen Lage und der konkreten Gefahr.

Durch die Annahme einer Aussetzung durch aktives Tun wird zugleich der von der Verteidigung im laufenden Verfahren herausgestrichenen Gefahr vorgebeugt, einem jeden Beamten die Pflicht aufzuerlegen, betrunkene Personen vorbeugend in Gewahrsam nehmen zu müssen, um dem Vorwurf einer Aussetzung zu begegnen. Der Umfang und das Bestehen einer Garantenpflicht gegenüber Betrunkenen bleiben somit weiter offen.

Die Ingewahrsamnahme des S wäre auch von $\$ 204$ I Nr. 1 LVwGSH gedeckt gewesen, da nach dieser Norm der Gewahrsam insbesondere zum Schutz von Personen gestattet ist, die sich auf Grund eines die freie Willenbestimmung ausschließenden Zustandes oder sonst in einer hilflosen Lage befinden. Gewahrsam nach \$204 I Nr. 1 LVwGSH und Aussetzung nach $\$ 221$ StGB knüpfen demnach an dieselbe Voraussetzung, die hilflose Lage, an. Immer wenn ein Beamter die hilflose Lage erkennt, ist zugleich die Möglichkeit des Gewahrsams eröffnet. Die vom Aussetzungstatbestand geforderten erheblichen Gefahren eröffnen unter Verhältnismäßigkeitsgesichtspunkten stets den Anwendungsbereich der Ingewahrsamnahme. Eine von der Verteidigung befürchtete Grauzone, in der die Beamten in jedem Fall ein Verfahren wegen Freiheitsberaubung oder Aussetzung zu befürchten hätten, besteht daher bei Annahme einer Aussetzung durch aktives Tun i.S.d. Nr. 1 nicht.

Den Schwerpunkt im Unterlassen der (weiteren) Hilfe erblicken zu wollen, behandelt den Sachverhalt dagegen nicht vollständig.

Wie sich bereits aus der Definition der hilflosen Lage ergibt, wird eine solche nur beendet, wenn hilfsfähige und hilfswillige Personen hinzukommen. Falls die Beamten S von vornherein nur aus ihrem Zuständigkeitsbereich hinausfahren wollten, waren sie zu keinem Zeitpunkt hilfswillig und S hätte sich auch im Polizeiauto in einer hilflosen Lage befunden. Eine Rettungschancenentziehung hätte nicht stattgefunden ${ }^{48}$. Die Rettungsmöglichkeiten in Groß Weeden, dem Ort, an dem $S$ aufgegriffen wurde, wären nicht besser oder schlechter gewesen als in Kronsforde, wo er zu Tode kam; die gleichen Temperaturen, ebenso wenig hilfsbereite Personen, der gleiche schlechte Zustand des S. Die Beamten hätten die Rettung daher schlicht unterlassen. Die Frage nach der Garantenstellung der Beamten für einen beliebigen Betrunkenen wäre zu problematisieren gewesen.

Da S aber zunächst in den Polizeigewahrsam gebracht werden sollte, hatte sich seine Lage verbessert und bildet so die notwendige Voraussetzung einer Aussetzung durch aktives Tun nach $\mathbb{2} 21$ I Nr. 1 StGB.

\section{Konkrete Lebens- oder schwere Gesundheitsgefahrl Gefährdungserfolg}

\section{a. Zu den Anforderungen an den Gefabrbegriff im Rabmen des $\int 221$ I StGB}

In Abgrenzung von der abstrakten Gefahr, die Voraussetzung der hilflosen Lage ist, muss sich die konkrete Gefahr gegenüber dieser „unmissverständlich profilieren“49. Der Eintritt einer konkret zugespitzten Gefahrenlage ist das entscheidende Einschränkungskriterium des objektiven Tatbestands und muss durch seinen Unrechtsgehalt den Strafrahmen von 3 Monaten bis zu 5 Jahren eröffnen. Keinesfalls dürfen die Anforderungen an die konkrete Gefahr daher zu gering $\operatorname{sein}^{50}$, wobei konkret erneut nichts anderes meint als eine bestimmte Wahrscheinlichkeit des Schadenseintritts.

Wie wahrscheinlich der Schadenseintritt sein muss, um von einer konkreten Gefahr sprechen zu können, lässt sich nun aber schwerlich in einer Prozentangabe ausdrücken, ganz abgesehen davon, dass eine solche Prozentangabe praktisch auch schwer oder überhaupt nicht zu bestimmen wäre ${ }^{51}$.

Es muss demnach eine natürliche Betrachtung Grundlage der Überlegungen sein.

Im Rahmen der Straßenverkehrsdelikte, die ebenfalls zu den konkreten Gefährdungsdelikten zählen, geht die nun herrschende Meinung davon aus, dass die Gefahr eines unmittelbaren Schadenseintritts bestanden haben muss und damit aufgrund einer objektiv nachträglichen Prognose die Sicherheit einer bestimmten Person im jeweiligen Moment so stark beeinträchtigt worden ist, dass es nur noch vom Zufall abhing, ob das Rechtsgut verletzt wurde oder nicht ${ }^{52}$. Meist wird dieser Zustand als „Beinaheunfall“ im Sinne einer zugespitzt kritischen Situation beschrieben ${ }^{53}$. Die abstrakte Gefahr wird demgegenüber als bloße Verallgemeinerung der aus Einzelfällen gewonnenen Erfahrungen definiert; die konkrete Gefahr muss jedoch auf Grundlage des jeweiligen Sachverhalts feststehen, ohne dass der Sachverhalt verallgemeinert oder abstrahiert wird ${ }^{54}$.

Eine zu großzügige Handhabung des Begriffs der Gefahr im Straßenverkehrsrecht in einigen früheren Entscheidungen des BGH, insbesondere im Hinblick auf die Gefährdung des Beifahrers ${ }^{55}$, wurde jeweils harsch kritisiert ${ }^{56}$. Insbesondere wurde wiederholt vor einer vom Einzelfall gelösten abstrakten Betrachtung der konkreten Gefahr gewarnt und eine größere Zurückhaltung bei ihrer Annahme gefordert ${ }^{57}$. Eine streng konkrete Betrachtungsweise ist erforderlich, um die Grenze zwischen abstrakten und konkreten Gefährdungsdelikten nicht zu verwischen und die $\mathbb{S} 315 \mathrm{ff}$. nicht durch Auslegung zu abstrakten Gefährdungsdelikten herunterzustilisieren. Dem ist der BGH für die Straßenverkehrsdelikte im Wesentlichen gefolgt ${ }^{58}$, wenn auch nicht so deutlich, wie von vielen erhofft. Das OLG Düsseldorf wollte beispielsweise nur eine „hochgradige Existenzkrise“ für das bedrohte Rechtsgut ausreichen lassen ${ }^{59}$.

Für die konkrete Gefahr im Rahmen des $\$ 221$ StGB können nun jedoch keinesfalls geringere Anforderungen gelten als bei den Stra- 
ßenverkehrsdelikten, da die Strafe im Gegensatz zu \315c StGB im Mindestmaß erhöht ist, ohne dass das Versetzen in eine hilflose Lage entsprechend der Trunkenheitsfahrt strafbewährt wäre.

Eine konkrete Gefahr liegt daher vor, wenn die Tathandlung über die ihr innewohnende Gefährlichkeit hinaus zu einer kritischen Situation für das geschützte Rechtsgut geführt hat, so dass eine Rettung nur noch vom Zufall abhing.

Die Auffassung von Jähnke, wonach sich der Gefahreneintritt in der Regel mit dem Zeitpunkt der Aussetzungshandlung deckt und es nicht auf die tatsächliche Steigerung der Schadenswahrscheinlichkeit und die situative Gefahr ankomme ${ }^{60}$, ist daher abzulehnen ${ }^{61}$.

Neben den Gefährdungsdelikten und einigen anderen strafrechtlichen Normen, wie der Notwehr, ist der Begriff der Gefahr insbesondere im Polizeirecht zentral. Die Gefahren werden dort weiter typisiert als abstrakt, konkret, erheblich, gegenwärtig oder dringend.

Eine konkrete Gefahr wird definiert als ein Zustand, in dem zum Zeitpunkt des Einschreitens objektive Anhaltspunkte dafür vorliegen, dass mit hinreichender Wahrscheinlichkeit in absehbarer Zeit bei ungestörtem Fortgang die Verletzung eines Schutzgutes eintritt und ex post gesehen, muss die Einschätzung zutreffend gewesen ein. Mit dem Eintritt der Verletzung muss daher ernsthaft zu rechnen sein. Die bloße theoretische Möglichkeit genügt nicht.

Erheblich ist eine Gefahr, wenn besonders schwere Rechtseinbußen drohen, im Falle des $\mathbb{2} 21$ StGB für das Leben oder für die körperliche Unversehrtheit in Form einer schweren Gesundheitsschädigung.

Eine gegenwärtige Gefahr ${ }^{62}$ besteht, wenn das schädigende Ereignis unmittelbar oder in allernächster Zukunft mit an Sicherheit grenzender Wahrscheinlichkeit bevorsteht bzw. die Schädigung bereits begonnen hat (zeitlich erhöhte Schadensnähe), und entspricht damit den Anforderungen eines Beinaheunfalls.

Ebel fordert daher auch im Rahmen des $₫ 221$ I StGB eine gegenwärtige Gefahr ${ }^{63}$, richtigerweise muss es sich sogar um eine erhebliche gegenwärtige und konkrete Gefahr handeln.

$\mathrm{Da}$ abstrakte Gefahren in nahezu jedem denkbaren Fall bestehen, muss in $\$ 221$ I StGB eine zugespitzte Gefahr gemeint sein, die den Strafrahmen rechtfertigt.

Über das Bestehen der Gefahr, die als solche nicht mehr als ein Wahrscheinlichkeitsurteil im Zustand der Unwissenheit zwischen dem Beginn eines Kausalgeschehens und dem Erfolgseintritt oder dessen Ausbleiben darstellt ${ }^{64}$, muss im Rahmen einer nachträglichen objektiven Prognose entschieden werden.

\section{b. Zum Vorliegen einer Gefahr im konkreten Fall}

Da $S$ tatsächlich überfahren wurde und an den Folgen des Unfalls gestorben ist, hat sich zumindest diese Gefahr realisiert. Die konkrete Gefahr des Überfahrenswerdens bestand aber erst ab dem Zeitpunkt, in dem S sich auf die Straße setzte und tatsächlich ein Auto Kurs auf ihn nahm. Hierauf muss sich im Folgenden der Gefährdungsvorsatz beziehen und auf nichts anderes!

Möglicherweise sah sich S aber neben der konkreten Gefahr des Überfahrenwerdens auch einer anderen konkreten Gefahr, nämlich dem Erfrierungstod ausgesetzt. Die Feststellungen im Urteil des Landgerichts Lübeck zu diesem Punkt sind unzureichend, wie ich später zeigen werde, aber von entscheidender Bedeutung.

Es liegt jedoch nahe, eine konkrete Gefahr für Leib und Leben des S auf Grund einer Unterkühlung anzunehmen.
Aufgrund des Wärmeregulationszentrums im Gehirn kann der menschliche Körper die Körpertemperatur nicht für unbegrenzte Zeit konstant bei den erforderlichen $37^{\circ} \mathrm{C}$ halten ${ }^{65}$. Wenn der Wärmeverlust bei Aufenthalt in der Kälte größer wird als die dem Körper mögliche Wärmeproduktion kommt es zur Unterkühlung.

Von allgemeiner Unterkühlung spricht man bei einer Körpertemperatur unter $35^{\circ} \mathrm{C}$. Ein Absinken der Körperkerntemperatur unter $26^{\circ} \mathrm{C}$ bis $28^{\circ} \mathrm{C}$ führt neben einer Erniedrigung der Stoffwechselvorgänge zur Atemlähmung sowie Herzschwäche (Herzrhythmusstörungen) und zum Todeseintritt. Besonders unterkühlungsgefährdet sind Menschen mit erhöhter Wärmeabgabe, vorrangig alkoholisierte Personen wie S, aber auch kleine Kinder, oder Menschen mit geminderter Wärmebildung, z.B. Erschöpfte, Kranke oder Hungernde. Feuchtigkeit und Wind begünstigen die Auskühlung des Körpers teilweise um ein Vielfaches. Durch ein Zusammentreffen dieser ungünstigen Umstände kann es bereits bei Temperaturen deutlich über dem Gefrierpunkt zum Kältetod kommen.

Eine Abnahme der Herzleistung und der Sauerstoffversorgung der Körpergewebe führt u. a. zu Hirnfunktionsstörungen, z.B. geminderter Kritikfähigkeit, Benommenheit oder Bewusstlosigkeit. Häufig kommt es bei einer Unterkühlung aber auch zu Sinnestäuschungen (Halluzinationen) und euphorischen Zuständen, was infolge eines paradoxen Wärmegefühls (Kälteidiotie) vor Eintritt der Bewusstlosigkeit dazu führt, dass sich Unterkühlte entkleiden. Ein Hinweis auf einen kurz bevorstehenden Kältetod könnte daher der Umstand gewesen sein, dass S sich die Schuhe auszog und auf die Straße setzte. Auch war $S$ bereits zuvor eine längere Zeit mit unzureichender Bekleidung der Kälte ausgesetzt gewesen und hatte eine nicht unerhebliche Strecke zu Fuß zurückgelegt. Ohne sachverständige Auskünfte oder weitere Sachverhaltsangaben lässt sich der nahe Erfrierungstod aber nicht sicher feststellen.

Das Urteil des BGH ist insoweit zu kritisieren, als die konkrete Gefahr, die dem Tatbestand der Aussetzung zugrunde gelegt werden soll, nicht benannt wird. Auch im Urteil des Landgerichts Kiel wird die möglicherweise drohende Erfrierung auf Seite 33 nicht weiter behandelt. Der Umstand, dass S seine Schuhe und Strümpfe ausgezogen hatte, blieb in allen Urteilen unkommentiert.

Es macht jedoch einen erheblichen Unterschied, welche konkrete Gefahr drohte, da genau diese Gefahr vom Vorsatz umfasst sein und sich im Rahmen der Erfolgsqualifikation realisieren muss. Keinesfalls kann daher offen bleiben, welche konkrete Gefahr gemeint ist.

\section{Kausalität zwischen hilfloser Lage und konkreter Gefahr}

Für den objektiven Tatbestand kann die Entscheidung, welche Gefahr eingetreten ist, jedoch zunächst offen bleiben, da sowohl die konkrete Gefahr, Opfer eines Verkehrsunfalls zu werden, als auch die konkrete Gefahr zu erfrieren, kausal auf der von den Beamten verursachten hilflosen Lage beruht. Der BGH führt insofern zutreffend aus, dass das verkehrsordnungswidrige Verhalten der Unfallverursacherin den Kausalzusammenhang nicht unterbrochen habe. Es sei anerkannt, dass eine Ursache im Rechtssinn ihre Bedeutung nicht verliere, wenn außer ihr noch andere Ursachen zur Herbeiführung des Erfolgs beitragen. Ein Ursachenzusammenhang sei nur dann zu verneinen, wenn ein späteres Ereignis die Fortwirkung der ursprünglichen Bedingung beseitige und seinerseits allein unter Eröffnung einer neuen Ursachenreihe den Erfolg herbeigeführt habe. $\mathrm{Zu}$ unterscheiden ist demnach zwischen den Fallgruppen der abge- 
brochenen und der fortwirkenden Kausalität. Der vorliegende Fall ist klar der fortwirkenden Kausalität zuzurechnen.

Auch die objektive Zurechnung muss bejaht werden.

Zweifel könnten sich allein daraus ergeben, dass die Todesursache durch eine dritte Person, die Autofahrerin, gesetzt wurde. Dass aber ein betrunkener und desorientierter Heranwachsender seinen Fußweg unter Umständen auf der Straße zurücklegt und nicht den Bürgersteig nutzt, liegt nicht außerhalb der Lebenswahrscheinlichkeit. Dass er dann aber möglicherweise von einem Auto erfasst wird, ist ebenfalls vorstellbar.

$S$ hat auch nicht eigenverantwortlich gehandelt, da er nicht im Vollbesitz seiner geistigen Kräfte war. Eine eigenverantwortliche Selbstgefährdung, die den Zurechnungszusammenhang durchbrechen würde, scheidet daher aus.

Mit diesen Feststellungen lässt sich problemlos der Fahrlässigkeitsvorwurf hinsichtlich $\$ 222$ StGB begründen.

\section{Anmerkungen zum subjektiver Tatbestand}

Der Täter muss aber auch in sein Bewusstsein aufgenommen haben, dass sein Verhalten zu einer bedrohlich zugespitzten Verschlechterung der Lage des Hilfsbedürftigen führen wird. Der Beinaheeintritt des Todes oder der schweren Gesundheitsschädigung als Entsprechung des Beinaheunfalls muss erkannt und gebilligt werden.

Insbesondere wenn Struensee von einer grotesken Regelung spricht und damit meint, dass im Falle des Abs. 3 eine fahrlässige Tötung, die sich von $\$ 222$ StGB nur durch den Gefährdungsvorsatz unterscheidet, den fast vollen Strafrahmen des vorsätzlichen Totschlags eröffnet, wird deutlich, dass der Gefährdungsvorsatz als qualifizierendes Moment dem Vorsatz hinsichtlich des Erfolgseintritts nahe und damit dem Tötungs- oder Verletzungsvorsatz nahe sein muss, ohne ihn gänzlich zu erreichen. Es muss daher bereits eine hohe Wahrscheinlichkeit für den Erfolgseintritt angenommen und gebilligt werden. An die Feststellungen zum subjektiven Tatbestand sind in einem Urteil ebenfalls hohe Anforderungen zu stellen. Nur so kann die Abgrenzung zwischen strafbarem Unrecht und einfachem Unglück gelingen. Der Begriff der Gefahr und der hierauf bezogene Vorsatz sind zur Begründung der Strafbarkeit von zentraler Bedeutung ${ }^{66}$.

Die Auffassung von Jähnke, dass der Täter keinen gedanklichen Schluss auf die durch die Aussetzung bewirkte Lebens- oder Leibesgefahr zu ziehen braucht ${ }^{67}$, scheint vor dem Hintergrund des weiten Tatbestands nahezu aberwitzig. Die von ihm zitierten BGH-Entscheidungen betreffen sämtlich nur die gefährliche Körperverletzung nach $\$ 224$ I Nr. 5 StGB mittels einer das Leben gefährdenden Behandlung, die bekanntlich abstrakt bestimmt wird.

$\mathrm{Zu}$ Recht fordern Wessels/Hettinger u.a. daher Vorsatz bezüglich der konkreten Gefahr ${ }^{68}$. Dieser kann entgegen der Ansicht von Horn ${ }^{69}$ auch vom Verletzungs- oder Tötungsvorsatz abgegrenzt werden, auch wenn er ihm nahe steht und im Regelfall mit diesem zusammentrifft. Der Unterschied liegt allein in der inneren Einstellung des Täters zum betroffenen Rechtsgut. So kann lediglich von einem Gefährdungsvorsatz gesprochen werden, wenn der Täter auf den Eintritt des rettenden Zufalls hofft, mag er auch noch so unwahrscheinlich sein. Insofern trifft ihn nach der herrschenden Billigungstheorie im Hinblick auf die Vollendung nur der Vorwurf bewusster Fahrlässigkeit.
Der Täter muss in Kenntnis der Verletzungsmöglichkeit in Kauf nehmen, eine Situation herbeizuführen, in der die Rechtsgutsverletzung wahrscheinlich ist, aber dennoch auf das Ausbleiben des Erfolgs vertrauen.

Das ist zum Beispiel der Fall, wenn eine hoffnungslos überforderte junge Mutter ihr Neugeborenes vor einem Krankenhaus aussetzt und dabei hofft, dass es vor dem Eintritt eines Kältetodes gefunden werde, obwohl sie sich nicht sicher ist und das Risiko bewusst eingeht.

Nach den Feststellungen des Landgerichts Lübeck wussten die Beamten, dass $S$ alkoholbedingt örtlich und situativ desorientiert war. Auch wussten sie, dass S für die herrschenden Temperaturen unzureichend bekleidet war. Diese Umstände waren ihnen zumindest im Sinne eines ständig aktualisierbaren Begleitwissens, häufig als sachgedankliches Mitbewusstsein bezeichnet, bekannt.

Feststellungen dazu, dass sich die Angeklagten aber auch nur im Entferntesten vorstellten, dass S überfahren werden könnte, fehlen jedoch vollständig. Lebensnah mag es sein, dass sie damit gerechnet haben, dass S aufgrund seiner Alkoholisierung erneut stürzt, sich möglicherweise bei einem erneuten Sturz verletzt, ja vielleicht auch auf Grund der niedrigen Temperaturen eine schwere Gesundheitsschädigung erleidet oder der Gefahr zu erfrieren ausgesetzt ist, aber dass sie sich vorstellten, $\mathrm{S}$ werde über den Grünstreifen, der den Bürgersteig von der Straße trennte, auf die Straße laufen und dort zumindest einen Beinaheunfall mit einem PKW erleiden, liegt nicht auf der Hand und bedürfte einer ausdrücklichen Erwähnung im Urteil. Es darf keinesfalls übersehen werden, dass der Vorsatz die konkrete Gefährdung erfassen muss, also das Herannahen des Autos. Ein Weniger würde nur im Rahmen einer bereits oben deutlich kritisierten und mit Entschiedenheit abzulehnenden abstrakten oder abstrahierenden Betrachtung genügen. Die Beamten haben sich aber wohl kaum Vorstellungen über die konkrete Möglichkeit eines potentiell tödlichen Autounfalls gemacht.

Nach den getroffenen Feststellungen könnte daher allenfalls ein Vorsatz bezüglich einer Unterkühlung mit all ihren Folgen bejaht werden, obwohl es auch insoweit an ausreichenden Feststellungen fehlt.

Falls noch keine konkrete Unterkühlungsgefahr gegeben gewesen wäre, müsste man daher sogar den Aussetzungstatbestand verneinen. Näher dürfte es aber liegen, dass eine konkrete Unterkühlungsgefahr bestanden hat und die Beamten dies auch billigend in Kauf nahmen.

Die Ausführungen des BGH können vor diesem Hintergrund also nicht überzeugen. Allein aus der Kenntnis der hilflosen Lage kann nicht auf das Erkennen der konkreten Gefahr, überfahren zu werden, und auch nicht auf die Billigung dieser Gefahr geschlossen werden. Die Feststellung „aus welchen Gründen sich die Angeklagten dann aber“ - gemeint ist hier nur die Kenntnis der hilflosen Lage! - „der konkreten Gefährdung des Lebens und der Gesundheit des Heranwachsenden nicht bewusst gewesen sein sollten, ist nicht ersichtlich “, genügt nicht, um einen wie auch immer gearteten Vorsatz zu begründen. Es fehlt an einer positiven Feststellung und damit an jeglicher Aussagekraft. Der BGH hat es damit versäumt, zur Frage des Gefährdungsvorsatzes sowie zur Frage des Unmittelbarkeitszusammenhangs bei nur hinsichtlich einer bestimmten Gefahr vorsätzlich verwirklichtem Grunddelikt Stellung zu beziehen. Welche Gefahr sich verwirklicht hat und ob hinsichtlich dieser konkreten Gefahr ein zumindest bedingter Vorsatz bestand, hätte der BGH und später das Landgericht Kiel deutlich herausstellen müssen. 
Auch im Urteil des Landgerichts Kiel auf Seite 14 wird nicht deutlich, dass es sich um eine konkrete Gefahr handeln muss, wenn es dort heißt: „hielt es jedenfalls der Angeklagte $\mathrm{M}$ für möglich, dass $\mathrm{S}$ aufgrund seines Zustandes nicht in der Lage sein würde, allein nach Hause oder in anderweitige Obhut zu gelangen, und dass ihm deshalb etwas zustoßen und er dadurch der Gefahr des Todes oder einer schweren Gesundheitsschädigung ausgesetzt sein könnte.“ Dass S einer Gefahr ausgesetzt sein „könnte“, ist lediglich die Beschreibung einer abstrakten Gefahr. Die Feststellungen zum subjektiven Tatbestand auf Seite 34 gehen ebenfalls nicht über den Vorsatz bezüglich einer abstrakten Gefahr hinaus. Der Vorsatz bezüglich der konkreten Gefahr wird entsprechend der Formulierung des BGH mit dem lapidaren Hinweis bejaht, es sei nicht ersichtlich, warum der Angeklagte keinen Vorsatz hinsichtlich der konkreten Gefahr gehabt haben sollte. Diese oberflächliche Herangehensweise verwundert insbesondere deshalb, weil das Landgericht Kiel, abgesehen vom Gefährdungsvorsatz und dem Unmittelbarkeitszusammenhang, den rechtlichen Schwerpunkten des Falls, sein Urteil auf insgesamt 43 Seiten dezidiert und nachvollziehbar begründet hat. Nur die beiden entscheidenden Passagen werden mit jeweils einem Satz abgehandelt.

Da die Formulierung des Landgerichts Kiel auch mit der Formulierung des BGH identisch ist, drängt sich fast schon der Verdacht auf, die rechtlichen Schwierigkeiten seien bemerkt worden, man wollte sich aber den eindeutigen Forderungen des BGH nicht widersetzen und hat stattdessen lieber einen Ausweg über eine geänderte Tatsachenfeststellung und den minder schweren Fall der Aussetzung gesucht.

Abschließend sei zum subjektiven Tatbestand angemerkt, dass jedenfalls kein Irrtum über den Kausalverlauf vorliegt, wenn sich der Täter eine bestimmte Gefahr vorstellt, aber eine andere Gefahr eintritt. Der Irrtum über den Kausalverlauf ist nämlich zu unterscheiden von einem schlichten Irrtum über den Taterfolg, hier die Art der konkreten Gefahr. Ein Irrtum über den Kausalverlauf setzt voraus, dass der gewollte Erfolg eintritt, nur auf andere und nicht vorhergesehene Art und Weise. Es liegt daher kein Irrtum über den Kausalverlauf vor, wenn ein anderer als der gewollte Erfolg eintritt. Der Vorsatz bezüglich einer Gefahr kann daher nicht durch den Vorsatz bezüglich einer anderen Gefahr ersetzt werden.

\section{Die Voraussetzungen des Qualifikationstatbestands nach \ 221 III StGB, insbesondere Unmittelbarkeitszu sammenhang}

Ausführungen zu Abs. 3 hat sich der BGH gespart, obwohl gerade die besonderen Merkmale der Erfolgsqualifikation nach hier vertretener Ansicht einer Bestrafung wegen Aussetzung mit Todesfolge entgegenstehen.

Strafschärfend wirkt sich nämlich nur die Realisierung „der von Absatz 1 erfassten Gefahren“ aus ${ }^{70}$. Bereits an dieser Stelle möchte ich daher ausdrücklich darauf hinweisen, dass es sich bei der realisierten Gefahr nur um eine solche handeln kann, die auch vom Vorsatz umfasst war.

Was bereits bei Sternberg-Lieben/Fisch anklingt, soll daher einmal deutlich herausgestellt werden.

Zur Verwirklichung des Grundtatbestandes muss sich der Täter eine oder mehrere konkrete Leibes- oder Lebensgefahren vorgestellt und diese gebilligt haben. Im Rahmen des Qualifikationstatbestandes können ihm nun aber nur solche Erfolge zugerechnet werden, hinsichtlich derer er den Grundtatbestand auch tatsächlich verwirklicht hat. Realisiert sich eine andere, vielleicht sogar eine andere typische Aussetzungsgefahr, die der Täter allerdings nicht bedacht oder nicht gebilligt hat, steht der Grundtatbestand in Tateinheit mit einer fahrlässigen Tötung nach $\$ 222$ StGB. In eine ähnliche Richtung zielt wohl auch Fischer, wenn er schreibt, die Eingrenzung des Qualifikationstatbestands könne durch eine restriktive Handhabung des Merkmals „durch die Tat“ erreicht werden ${ }^{71}$.

Bezüglich der Realisierung der vorsätzlich verursachten und deliktstypischen Gefahren des Abs. 1 genügt dann allerdings gemäß $\$ 18$ StGB jede Form der Fahrlässigkeit.

Unstreitig beruht der Tod des S kausal auf der Aussetzungshandlung. Neben die Kausalität im Sinne der Conditio-sine-qua-non-Formel tritt bei den erfolgsqualifizierten Delikten wegen des im Vergleich zum Grund- und Fahrlässigkeitsdelikt wesentlich erhöhten Strafrahmens allerdings der so genannte Unmittelbarkeitszusammenhang als Kriterium der objektiven Zurechnung. Die vom Grunddelikt ausgehende spezifische Gefahr muss sich danach ohne wesentliche Zwischenschritte in dem konkret eingetretenen qualifizierenden Erfolg niedergeschlagen haben, d.h. die Gefährlichkeit des Grunddelikts selbst - und nicht ein anderes Gefährdungsmoment - muss den Erfolg verursacht haben.

Realisiert hat sich vorliegend eine Verkehrsgefahr. Die Anforderungen an die konkrete Gefahr müssen daher der des $\$ 315$ c StGB entsprechen.

Die Beamten stellten sich aber keinen Beinaheunfall, d.h. keine konkrete Gefahr des Überfahrenwerdens vor. Allein die Gefahr einer Unterkühlung könnte vom Vorsatz umfasst gewesen sein. Hinsichtlich der verwirklichten Gefahr haben sie das Grunddelikt nicht verwirklicht. An dieser Stelle wird nun deutlich, warum es für einen angemessenen Umgang mit $\$ 221$ StGB von so zentraler Bedeutung ist, die Gefahr und den entsprechenden Vorsatz genau zu bezeichnen. Unter dem Gesichtspunkt der Schuldangemessenheit kann nämlich nicht jede fahrlässige Tötung, die mit einer Aussetzung tateinheitlich zusammentrifft, eine Aussetzung mit Todesfolge darstellen. Freiheitsstrafe nicht unter 3 Jahren ist nur dann tat- und schuldangemessen, wenn sich die vom Täter in Kauf genommene Gefahr realisiert und nicht eine andere Gefahr, wie im vorliegenden Fall.

Zwischen dem Grundtatbestand und damit der Unterkühlungsgefahr und dem Unfalltod fehlt es daher an dem spezifischen Gefahrzusammenhang.

In Betracht kommt daher allein eine vollendete Aussetzung in Tateinheit mit einer fahrlässigen Tötung. Das Unrecht der Tat spiegelt sich in diesem Ergebnis adäquat wider und zugleich werden die zwingenden beamtenrechtlichen Folgen der $\mathbb{\int} 48$ ff. BBG und der entsprechenden landesrechtlichen Regelungen vermieden. Auch wird den Genugtuungsinteressen der Hinterbliebene umfassend Rechnung getragen, da sowohl das vorsätzliche Fehlverhalten der Beamten, als auch der schmerzliche Verlust des Sohnes geahndet werden.

Weitere disziplinarrechtliche Konsequenzen, durch die ein ähnlich unprofessionelles Vorgehen der Angeklagten in Zukunft vermieden werden kann, runden das Ergebnis ab.

Die bloße Behauptung des Landgerichts Kiel auf Seite 37, in dem Tod habe sich auch die durch die Aussetzung geschaffene spezifische Gefahr verwirklicht, ohne zuvor festzustellen, welche Gefahr vom Vorsatz umfasst war, ist in jeder Hinsicht unzureichend. 


\section{B. Fazit}

Nach Fischer können Spannungen, die durch die hohe Mindeststrafe des Abs. 3 eintreten, nur im Gnadenwege beseitigt werden ${ }^{72}$. Dem ist nicht zuzustimmen. Der Gnadenweg über $\ 50$ BBG oder die entsprechenden Regelungen für Landesbeamte kann nur der letzte Ausweg sein. Gleiches gilt für die Annahme eines minder schweren Falls.

Vielmehr zwingt die hohe Strafdrohung auch insoweit zu einer restriktiven Auslegung schon des Grundtatbestands. Wenn ein Strafrahmen erkennbar höheres Unrecht pönalisiert, als die Fälle beinhalten, die der Richter unter die Norm subsumieren will, muss der Straftatbestand zwingend restriktiv ausgelegt werden. Es darf nicht der Umweg über einen minder schweren Fall gewählt werden, da dieser die Bewertung der Tat als Verbrechen nach $\$ 12$ StGB unberührt lässt und an die Einstufung einer Tat als Verbrechen zahlreiche rechtliche Konsequenzen geknüpft sind, nicht nur im Bereich des Beamtenrechts, sondern auch für den Versuch, die versuchte Anstiftung nach $\ 30$ StGB, die Sicherungsverwahrung und einige mehr.

Taugliche Anknüpfungspunkte für eine restriktive Auslegung des Aussetzungstatbestands dürften nach dem eben gesagten die Beschränkung auf aussetzungstypische Gefahren, hohe Anforderungen an die konkrete Gefahr und den hierauf bezogenen Vorsatz sowie die Formulierung „durch die Tat“ in Abs. 3 und die daraus folgenden Konsequenzen für den Unmittelbarkeitszusammenhang darstellen. Eine solche restriktive Auslegung ist wegen der hohen Mindeststrafe in Abs. 1 und 3 auch zwingend geboten.

\section{Fußnoten:}

* Der Autor ist wissenschaftlicher Mitarbeiter am Institut für Sanktionenrecht und Kriminologie der Christian-Albrechts-Universität zu Kiel unter der Leitung von Prof. Dr. Monika Frommel.

1 Frommel, 40 Jahre Strafrechtsreform, NK 2008, 133 (133).

2 Frommel, 40 Jahre Strafrechtsreform, NK 2008, 133 (134).

3 Vgl. Hinz, DRiZ 2001, 321 (321).

4 Vgl. zur Wandlung eines vom Resozialisierungsgedanken beherrschten, täterzentrierten Strafrechtssystems hin zu einer stärkeren Opferorientierung Hinz, DRiZ 2001, 321 (324).

5 Gesetz zur Verbesserung der Rechte von Verletzten im Strafverfahren vom 24.06.2004.

6 Zweites Gesetz zur Modernisierung der Justiz vom 22.12.2006.

7 Vgl. zu weiteren durch das Opferrechtsreformgesetz eingeführten Neuerungen Ferber, NJW 2004, 2562 ff.

8 Eingeführt durch das zweites Gesetz zur Modernisierung der Justiz vom 22.12.2006.

9 Pressemitteilung des BMJ, abzurufen unter: http://www.bmj.bund.de/enid/ Pressestelle/Pressemitteilungen_58.html?druck $=1 \&$ pmc_id=5561, Stand 21.01.2009.

10 Pressemitteilung des BMJ, abzurufen unter: http://www.bmj.bund.de/enid/ Pressestelle/Pressemitteilungen_58.html?druck=1\&pmc_id=5561, Stand 21.01.2009.

11 Vgl. zu entsprechenden Forderungen Heinz, NK 2008, 50 ff.; ders., Vortrag vom 31.03.2007, abzurufen unter http://www.uni-konstanz.de/rtf/ kis/Heinz_Mehr_und_haertere_Strafen_he306.pdf; Kersten, NK 2008, 49 (49).

12 Historisch betrachtet hat die Viktimodogmatik unter dem Blickwinkel der Eigenverantwortlichkeit des Opfers eher zu einer Einschränkung der Verantwortlichkeit des Täters geführt. Der Begriff erfasst allerdings auch jede Form der erweiternden Auslegung unter spezifischer Berücksichtigung von Opferbelangen.

13 Z.B. Frommel, NK 2003, 46 (47).

$14 \mathrm{Zu}$ Sinn und Zweck des Strafens unter viktimologischen Gesichtspunkten sei an dieser Stelle ausdrücklich auf den lesenswerten Artikel von Hinz, DRiZ 2001, 321 ff., hingewiesen.

15 Reemtsma in: Hassemer/Reemtsma, Verbrechensopfer, Gesetz und Gerechtigkeit, 2002, Kapitel 6, Das Interesse des Opfers am strafenden Staat, 112 (131); ders., Im Keller, 1998, 216.

16 Vgl. zu den in der Literatur gelegentlich geäußerten Bedenken Hinz, DRiZ 2001, 321 (326 f.) mit entsprechenden Nachweisen.

17 Teilweise abgedruckt in NStZ 2008, $395 \mathrm{f}$.

18 Entscheidung bisher unveröffentlicht.
19 So auch Hardtung in: MüKo, 2003, $\mathbb{S} 221$ Rn. 5 m.w.N.; a.A. Jähnke in: LK-StGB, 11. Aufl. 2005, $\mathbb{2} 21$ Rn. 7, 18.

20 Ebel, NStZ 2002, 404 (404).

21 Anmerkung des Verfassers.

22 Hardtung, o. Fn. 19, \221 Rn. 7; BGH, Urteil vom 10. Januar - 3 StR 463/07.

23 Hardtung, o. Fn. 19, $\$ 221$ Rn. 5

24 Hardtung, o. Fn. 19, $\mathbb{S} 221$ Rn. 5.

25 Hardtung, o. Fn. 19, \ 221 Rn. 6.

26 Vertiefend Ebel, NStZ 2002, 404 (407); ebenso Hacker/Lautner, Jura 2006, $274(274)$.

27 Vg. Ebel, NStZ 2002, 404 (407).

28 BGH, NStZ-RR 1998, 7 f. und 187 f. m.w.N.

29 BGH, NStZ-RR 1998, 7 f. und 187 f. m.w.N.

30 Hardtung, o. Fn. 19, \$2 221 Rn. 3.

31 Hacker/Lautner, Jura 2006, 274 (276) m.w.N.

32 Fischer, StGB-Kommentar, 55. Aufl. (2008), $\$ 221$ Rn. 7.

33 Hardtung, o. Fn. 19, $\mathbb{} 221$ Rn. 12.

34 So auch Hardtung, o. Fn. 19, \ 221 Rn. 12.

35 Ähnlich Struensee in: Dencker/Struensee/Nelles/Stein, Einführung in das 6. Strafrechtsreformgesetz, 1998, Rn. 23 m.w.N.; er fordert, dass es in der jeweiligen Situation nur noch vom Zufall abhängt, ob das Opfer gerettet wird oder nicht.

36 So z.B. Hardtung, o. Fn. 19, \$221 Rn. 18.

37 Sternberg-Lieben/Fisch, Jura 1999, 45 (46).

38 Ähnlich aber Ebel, NStZ 2002, 404 (408), der auf „kurze fürchterliche Sekunden“ und die „Verzweiflung“ des Ausgesetzten abstellt.

39 So auch Sternberg-Lieben/Fisch, Jura 1999, 45 (45 f.).

40 So aber Ebel, NStZ 2002, 404 (407) m.w.N.

41 So aber Fischer, o. Fn. 32, $\mathbb{} 221$ Rn. 1; Jähnke, o. Fn. 19, $\mathbb{} 221$ Rn. 1; kritisch Struensee, o. Fn. 35, Rn. 22, 27 ff. und 32 f.; ablehnend Ebel, NStZ 2002, 404 (404).

42 Hardtung, o. Fn. 19, $\mathbb{2} 21$ Rn. 8.

43 Sternberg-Lieben/Fisch, Jura 1999, 45 (46).

44 BGHSt 6, 46 (59); Wessels/ Beulke, AT, 38. Aufl. 2008, Rn. 700; Schönke/ Schröder - Stree, 27. Aufl. 2006, Vor $\$ \mathbb{S} 13$ ff. Rn. 158.

45 Vgl. zur Voraussetzung der gesicherten Lage Sternberg-Lieben/Fisch, Jura 1999, 45 (46).

46 KG, JR 1973, 72.

47 Auch zwischen der Versetzungshandlung und der hilflosen Lage muss Kausalität bestehen und die hilflose Lage muss dem Täter objektiv zurechenbar sein; vgl. hierzu Ebel, NStZ 2002, 404 (406).

48 Vgl. zum Begriff Sternberg-Lieben/Fisch, Jura 1999, 45 (47) m.w.N.

49 So Ebel, NStZ 2002, 404 (405).

50 Ebel, NStZ 2002, 404 (405).

51 Gegen eine Prozentangabe auch BGHSt 18, $271 \mathrm{ff}$.

52 BGH, NStZ-RR 1997, 200; NJW 1995, 3131 (3132).

53 BGH, NJW 2003, 128, NStZ-RR 1997, 200; NJW 1995, 3131 (3132); Lackner/Kühl, StGB, 26. Aufl. 2007, \$221 Rn. 5; Hacker/Lautner, Jura 2006, 275 (276).

54 BayObLG, NJW 1990, $133 \mathrm{f}$.

55 Vgl. beispielhaft BGH, NJW 1989, 1227.

56 BayObLGSt 1988, 76 ff. m.w.N; BayOblG, NJW 1990, 133.

57 Berz, NVZ 1989, 409 ff.; Hauf, DAR 1994, 59 ff.

58 BGH, NStZ-RR 1997, 200; NJW 1995, 3131.

59 OLG Düsseldorf, NJW 1993, 3212.

60 Jähnke, o. Fn. 10, \$221 Rn. 19; OLG Zweibrücken, NStZ 1997601 (602).

61 Hacker/Lautner, Jura 2006, 274 (275) mit weitergehenden Argumenten.

62 Vgl. zur Gegenwärtigkeit im Strafrecht Fischer, o. Fn. 32, $\$ 32$ Rn. 17 f.

63 Ebel, NStZ 2002, 404 (405).

64 Zum Begriff der Gefahr und ihrem Ursprung in der Unwissenheit des Menschen sehr instruktiv Struensee, o. Fn. 35, Rn. 14 und 18 ff.

65 Die folgenden Informationen sind der Homepage des rechtsmedizinischen Instituts der Universität Leipzig entnommen, http://rechtsmedizin.uni-leipzig.de/home/content/wissen_a_z/kaeltetod/index.htm, Stand 21.01.2009; zur Vertiefung wird der Aufsatz von Madea/Preuß/Lignitz, Unterkühlung, Rechtsmedizin 2004, Seite 41 bis 57, empfohlen. Online abzurufen unter http://www.springerlink.com/content/yb67gffl0jxufmuh/fulltext.pdf, Stand 21.01.2009.

66 Struensee, o. Fn. 35, Rn. 12.

67 Jähnke, o. Fn. 19, \221 Rn. 36.

68 Wessels/Hettinger, BT 1, 32. Aufl. 2008, Rn. 205 m.w.N.

69 Horn, Konkrete Gefährdungsdelikte, 1973, 204 ff.; ebenso Wolters/Horn in: SK-StGB, 65. Lfg. 2006, Vor $\mathbb{3} 306, \mathrm{Rn} .14$.

70 So Sternberg-Lieben/Fisch, Jura 1999, 45 (50).

71 Fischer, o. Fn. 32, 221 Rn. 17.

72 Fischer, StGB-Kommentar, 56. Aufl. (2009), $\$ 221$ Rn. 28. 